PROCEEDINGS OF THE

AMERICAN MATHEMATICAL SOCIETY

Volume 136, Number 7, July 2008, Pages 2515-2520

S 0002-9939(08)09193-4

Article electronically published on March 5, 2008

\title{
HUREWICZ SETS OF REALS WITHOUT PERFECT SUBSETS
}

\author{
DUŠAN REPOVŠ, BOAZ TSABAN, AND LYUBOMYR ZDOMSKYY
}

(Communicated by Julia Knight)

\begin{abstract}
We show that even for subsets $X$ of the real line that do not contain perfect sets, the Hurewicz property does not imply the property $S_{1}(\Gamma, \Gamma)$, asserting that for each countable family of open $\gamma$-covers of $X$, there is a choice function whose image is a $\gamma$-cover of $X$. This settles a problem of Just, Miller, Scheepers, and Szeptycki. Our main result also answers a question of Bartoszyński and the second author, and implies that for $C_{p}(X)$, the conjunction of Sakai's strong countable fan tightness and the Reznichenko property does not imply Arhangel'skiı̌'s property $\alpha_{2}$.
\end{abstract}

\section{INTRODUCTION}

By a set of reals we mean a separable, zero-dimensional, and metrizable space (such spaces are homeomorphic to subsets of the real line $\mathbb{R}$ ). Fix a set of reals $X$. Let $\mathcal{O}$ denote the collection of all open covers of $X$. An open cover $\mathcal{U}$ of $X$ is a $\gamma$-cover of $X$ if it is infinite and for each $x \in X, x$ is a member of all but finitely many members of $\mathcal{U}$. Let $\Gamma$ denote the collection of all open $\gamma$-covers of $X$. Motivated by Menger's work, Hurewicz [6 introduced the Hurewicz property $\mathrm{U}_{\text {fin }}(\mathcal{O}, \Gamma)$ :

For each sequence $\left\{\mathcal{U}_{n}\right\}_{n \in \mathbb{N}}$ of members of $\mathcal{O}$ that do not contain a finite subcover, there exist finite sets $\mathcal{F}_{n} \subseteq \mathcal{U}_{n}, n \in \mathbb{N}$, such that $\left\{\bigcup \mathcal{F}_{n}: n \in \mathbb{N}\right\} \in \Gamma$.

Every $\sigma$-compact space satisfies $\mathrm{U}_{\text {fin }}(\mathcal{O}, \Gamma)$, but the converse fails [7, 2 .

Let $\mathscr{A}$ and $\mathscr{B}$ be any two families. Motivated by works of Rothberger, Scheepers introduced the following prototype of properties [12:

$\mathrm{S}_{1}(\mathscr{A}, \mathscr{B})$ : For each sequence $\left\{\mathcal{U}_{n}\right\}_{n \in \mathbb{N}}$ of members of $\mathscr{A}$, there exist members $U_{n} \in \mathcal{U}_{n}, n \in \mathbb{N}$, such that $\left\{U_{n}: n \in \mathbb{N}\right\} \in \mathscr{B}$.

It is easy to see that $\mathrm{U}_{\text {fin }}(\mathcal{O}, \Gamma)=\mathrm{U}_{\text {fin }}(\Gamma, \Gamma)$, and therefore $\mathrm{S}_{1}(\Gamma, \Gamma)$ implies $\mathrm{U}_{\text {fin }}(\mathcal{O}, \Gamma)$ [12. However, a set of reals satisfying $S_{1}(\Gamma, \Gamma)$ cannot contain perfect subsets 7 . It follows that, for example, $\mathbb{R}$ satisfies $\bigcup_{\text {fin }}(\mathcal{O}, \Gamma)$ but not $S_{1}(\Gamma, \Gamma)$. In the fundamental paper [7, we are asked whether there are nontrivial examples showing that $\mathrm{U}_{\text {fin }}(\mathcal{O}, \Gamma)$ does not imply $\mathrm{S}_{1}(\Gamma, \Gamma)$.

Received by the editors December 5, 2006, and, in revised form, April 9, 2007.

2000 Mathematics Subject Classification. Primary 37F20; Secondary 26A03, 03E70.

The first and the third authors were supported by the Slovenian Research Agency grants P1-0292-0101-04 and BI-UA/04-06-007.

The second author was partially supported by the Koshland Center for Basic Research. 
Problem 1.1 (Just, Miller, Scheepers, Szeptycki [7). Let $X$ be a set of reals that does not contain a perfect set, but that does have the Hurewicz property. Does $X$ then satisfy $S_{1}(\Gamma, \Gamma)$ ?

We give a negative answer that also yields a new result concerning function spaces.

\section{The MAIN THEOREM}

We prove a stronger assertion than what is needed to settle Problem 1.1 this will be useful for the next section. Let $C_{\Gamma}$ denote the collection of all clopen $\gamma$-covers of $X$. Clearly, $\mathrm{S}_{1}(\Gamma, \Gamma)$ implies $\mathrm{S}_{1}\left(C_{\Gamma}, C_{\Gamma}\right)$ 1] The hypothesis in the following theorem is a consequence of the Continuum Hypothesis. See 3 for a survey of the involved cardinals.

Theorem 2.1. Assume that $\mathfrak{b}=\mathfrak{c}$. There exists a set of reals $X$ such that:

(1) $X$ does not contain a perfect set;

(2) all finite powers of $X$ have the Hurewicz property $\mathrm{U}_{\text {fin }}(\mathcal{O}, \Gamma)$;

(3) no set of reals containing $X$ satisfies $\mathrm{S}_{1}\left(C_{\Gamma}, C_{\Gamma}\right)$.

Theorem 2.1 is proved in three steps. The first step is analogous to Theorem 4.2 of [5] and will be used to show that the constructed set is not contained in a set of reals satisfying $\mathrm{S}_{1}\left(C_{\Gamma}, C_{\Gamma}\right)$. We say that a convergent sequence $\left\{x_{n}\right\}_{n \in \mathbb{N}}$ is nontrivial if $\lim _{n} x_{n} \notin\left\{x_{n}: n \in \mathbb{N}\right\}$.

Lemma 2.2. Let $X$ be a subspace of a zero-dimensional metrizable space $Y$ satisfying $\mathrm{S}_{1}\left(C_{\Gamma}, C_{\Gamma}\right)$, and let $\left\{x_{n}^{m}\right\}_{n \in \mathbb{N}}, m \in \mathbb{N}$, be nontrivial convergent sequences in $X$. Then there are a countable closed cover $\left\{F_{k}: k \in \mathbb{N}\right\}$ of $X$ and an infinite $A \subseteq \mathbb{N}$, such that $F_{k} \cap\left\{x_{n}^{m}: n \in A\right\}$ is finite for all $k, m$.

Proof. Let $d$ be a metric on $Y$ that generates its topology. For each $m$, do the following. Let $x_{m}=\lim _{n} x_{n}^{m}$, and for each $n$ take a clopen neighborhood $C_{n}^{m}$ of $x_{n}^{m}$ in $Y$, whose diameter is smaller than $d\left(x_{n}^{m}, x_{m}\right) / 2$. For each $m, n$, set

$$
U_{n}^{m}=Y \backslash\left(C_{n}^{0} \cup C_{n}^{1} \cup \cdots \cup C_{n}^{m}\right) .
$$

For each $m,\left\{U_{n}^{m}: n \in \mathbb{N}\right\}$ is a clopen $\gamma$-cover of $Y$. Apply $\mathrm{S}_{1}\left(C_{\Gamma}, C_{\Gamma}\right)$ to get $f \in \mathbb{N}^{\mathbb{N}}$ such that $\left\{U_{f(m)}^{m}: m \in \mathbb{N}\right\}$ is a (clopen) $\gamma$-cover of $Y$. As $U_{f(m)}^{m} \subseteq Y \backslash C_{f(m)}^{0}$ for each $m$, we have that the image $A$ of $f$ is infinite.

For each $k$, let $F_{k}=\bigcap_{i \geq k} U_{f(i)}^{i} .\left\{F_{k}: k \in \mathbb{N}\right\}$ is a closed $(\gamma-)$ cover of $Y$. Fix $k$ and $m$. If $n$ is large enough and $n \in A$, then $n=f(i)$ with $i \geq m, k$. As $x_{n}^{m}=x_{f(i)}^{m} \in C_{f(i)}^{m}$ and $i \geq m, x_{n}^{m} \notin U_{f(i)}^{i}$. As $i \geq k, U_{f(i)}^{i} \supseteq F_{k}$, and therefore $x_{n}^{m} \notin F_{k}$.

To make sure that our constructed set does not contain a perfect set and that it satisfies the Hurewicz property in all finite powers, we will use the following. Let $\overline{\mathbb{N}}=\mathbb{N} \cup\{\infty\}$ be the one-point compactification of $\mathbb{N}$, and $\overline{\mathbb{N}}^{\uparrow \mathbb{N}}$ be the collection of all nondecreasing elements $f$ of $\overline{\mathbb{N}}^{\mathbb{N}}$ (endowed with the Tychonoff product topology) such that $f(n)<f(n+1)$ whenever $f(n)<\infty . \overline{\mathbb{N}}^{\uparrow \mathbb{N}}$ is homeomorphic to the Cantor space (see [13] for an explicit homeomorphism) and can therefore be viewed as a set of reals.

\footnotetext{
${ }^{1}$ It is an open problem whether the converse implication holds [4, 11].
} 
Let $S$ be the family of all nondecreasing finite sequences in $\mathbb{N}$. For $s \in S,|s|$ denotes its length. For each $s \in S$, define $q_{s} \in \overline{\mathbb{N}}^{\uparrow \mathbb{N}}$ by $q_{s}(n)=s(n)$ if $n<|s|$, and $q_{s}(n)=\infty$ otherwise. Let $Q$ be the collection of all these elements $q_{s} . Q$ is dense in $\overline{\mathbb{N}}^{\uparrow}$.

For a set $D$ and $f, g \in \mathbb{N}^{D}, f \leq^{*} g$ means: $f(d) \leq g(d)$ for all but finitely many $d \in D$. A $\mathfrak{b}$-scale is an unbounded (with respect to $\leq^{*}$ ) set $\left\{f_{\alpha}: \alpha<\mathfrak{b}\right\} \subseteq \mathbb{N}^{\mathbb{N}}$ of increasing functions, such that $f_{\alpha} \leq^{*} f_{\beta}$ whenever $\alpha<\beta$.

Theorem 2.3 (Bartoszyński-Tsaban [2]). Let $X \subseteq \overline{\mathbb{N}}^{\uparrow \mathbb{N}}$ be a union of a $\mathfrak{b}$-scale and $Q$. Then $X$ contains no perfect subset, and all finite powers of $H$ satisfy the Hurewicz property $\mathrm{U}_{\text {fin }}(\mathcal{O}, \Gamma)$.

For each $s \in S,\left\{q_{s^{\wedge} n}\right\}_{n \in \mathbb{N}}$ (where ^ denotes a concatenation of sequences) is a nontrivial convergent sequence in $\overline{\mathbb{N}}^{\uparrow \mathbb{N}} 2$ and

$$
\lim _{n \rightarrow \infty} q_{s^{\wedge} n}=q_{s} .
$$

The following will be used in our construction.

Lemma 2.4. Let $X$ be a closed subspace of $\overline{\mathbb{N}}^{\uparrow \mathbb{N}}$. If $X \cap\left\{q_{s^{\wedge} n}: n \in \mathbb{N}\right\}$ is finite for each $s \in S$, then there exists $\phi: S \rightarrow \mathbb{N}$ such that for all $x \in X$ and all $n \geq 2$, $x(n) \geq \phi(x \uparrow n)$ implies $x(n+1) \leq \phi(x \uparrow(n+1))$.

Proof. For each $s \in S$, let $k(s)$ be such that $q_{s^{\wedge} k} \in \overline{\mathbb{N}}^{\uparrow \mathbb{N}} \backslash X$ for all $k \geq k(s)$. As $X$ is closed in $\overline{\mathbb{N}}^{\uparrow \mathbb{N}}$, for each $k \geq k(s)$ there is $m(s, k)$ such that

$$
\left\{z \in \overline{\mathbb{N}}^{\uparrow \mathbb{N}}: z \uparrow(|s|+1)=s^{\wedge} k, z(|s|+1)>m(s, k)\right\} \cap X=\emptyset .
$$

(Note that $\left\{z \in \overline{\mathbb{N}}^{\uparrow \mathbb{N}}: z \uparrow(|s|+1)=s^{\wedge} k, z(|s|+1)>m\right\}, m \in \mathbb{N}$, is a neighborhood base at $q_{s^{\wedge} k}$.) Define $\phi: S \rightarrow \mathbb{N}$ by

$$
\phi(s)=\max \{k(s), m(s \uparrow(|s|-1)), s(|s|-1)\}
$$

when $|s| \geq 2$, and by $\phi(s)=0$ when $|s|<2$. Let $x \in X$ and $n \geq 2$. If $x(n) \geq$ $\phi(x\lceil n)$, then $x(n) \geq k(x\lceil n)$; hence $x(n+1) \leq m(x \uparrow n, x(n)) \leq \phi(x \uparrow(n+1))$.

It remains to prove the following.

Proposition 2.5. Assume that $\mathfrak{b}=\mathfrak{c}$. There exists a $\mathfrak{b}$-scale $B=\left\{b_{\alpha}: \alpha<\mathfrak{b}\right\}$ such that for each closed cover $\left\{F_{n}: n \in \mathbb{N}\right\}$ of $B \cup Q$ and each infinite set $A \subseteq \mathbb{N}$, there are $n$ and $s \in S$ such that $F_{n} \cap\left\{q_{s^{\wedge} k}: k \in A\right\}$ is infinite.

Proof. Let $\left\{A_{\alpha}: \alpha<\mathfrak{c}\right\}$ be an enumeration of all infinite subsets of $\mathbb{N}$, such that for each infinite $A \subseteq \mathbb{N}$, there are $\mathfrak{c}$ many $\alpha<\mathfrak{c}$ with $A_{\alpha}=A$.

As $\mathfrak{b}=\mathfrak{d}=\mathfrak{c}$, there is a (standard) scale in $\mathbb{N}^{S}$, that is, a family $\left\{\phi_{\alpha}: \alpha<\mathfrak{c}\right\} \subseteq$ $\mathbb{N}^{S}$ such that:

(1) for each $\phi \in \mathbb{N}^{S}$, there is $\beta<\mathfrak{c}$ such that $\phi \leq^{*} \phi_{\beta}$;

(2) for all $\alpha<\beta<\mathfrak{c}, \phi_{\alpha} \leq^{*} \phi_{\beta}$.

\footnotetext{
${ }^{2}$ Strictly speaking, $q_{s^{\wedge} n} \notin \overline{\mathbb{N}}^{\uparrow \mathbb{N}}$ when $n<s(|s|-1)$, but since we are dealing with convergent sequences, we can ignore the first few elements.
} 
For an infinite $A \subseteq \mathbb{N}$, let $\bar{A}=A \cup\{\infty\}$ and

$$
\bar{A}^{\uparrow \mathbb{N}}=\left\{x \in \overline{\mathbb{N}}^{\uparrow \mathbb{N}}: x(n) \in \bar{A} \text { for all } n\right\} .
$$

The order isomorphism between $A \cup\{\infty\}$ and $\mathbb{N} \cup\{\infty\}$ induces an order isomorphism $\Psi_{A}: \bar{A}^{\uparrow \mathbb{N}} \rightarrow \overline{\mathbb{N}}^{\uparrow \mathbb{N}}$.

By induction on $\alpha<\mathfrak{b}=\mathfrak{c}$, construct a $\mathfrak{b}$-scale $B=\left\{b_{\alpha}: \alpha<\mathfrak{c}\right\}$ such that for each $\alpha<\mathfrak{c}, b_{\alpha} \in\left(A_{\alpha}\right)^{\uparrow \mathbb{N}}$, and

$$
\Psi_{A_{\alpha}}\left(b_{\alpha}\right)(n)>\phi_{\alpha}\left(\Psi_{A_{\alpha}}\left(b_{\alpha}\right)\lceil n)\right.
$$

for all $n \geq 2$.

We claim that $X=B \cup Q$ is as required. Indeed, let $A$ be an infinite subset of $\mathbb{N}$. Take an increasing enumeration $\left\{\beta_{\alpha}: \alpha<\mathfrak{c}\right\}$ of $\left\{\alpha<\mathfrak{c}: A_{\alpha}=A\right\}$. For each $\alpha<\mathfrak{c}, b_{\beta_{\alpha}} \in \bar{A}^{\uparrow \mathbb{N}}$. Set $c_{\alpha}=\Psi_{A}\left(b_{\beta_{\alpha}}\right)$, and $C=\left\{c_{\alpha}: \alpha<\mathfrak{c}\right\}$. By the construction of the functions $b_{\alpha}$,

$$
c_{\alpha}(n)>\phi_{\beta_{\alpha}}\left(c_{\alpha}\lceil n) \geq \phi_{\alpha}\left(c_{\alpha}\lceil n)\right.\right.
$$

for all but finitely many $n$.

Let $\left\{K_{m}: m \in \mathbb{N}\right\}$ be a closed cover of $C \cup Q$. Then there are $m$ and $s \in S$ such that $K_{m} \cap\left\{q_{s^{\wedge} k}: k \in \mathbb{N}\right\}$ is infinite: Otherwise, by Lemma 2.4, for each $m$ there is $\psi_{m} \in \mathbb{N}^{S}$ such that for all $x \in K_{m}$ and $n \geq 2, x(n) \geq \psi_{m}(x\lceil n)$ implies $x(n+1) \leq \psi_{m}\left(x\lceil(n+1))\right.$. Let $\alpha<\mathfrak{c}$ be such that for each $m, \phi_{\alpha}(s) \geq \psi_{m}(s)$ for all but finitely many $s \in S$. It is easy to verify that $c_{\alpha} \notin K_{m}$ for all $m$, a contradiction.

Now consider any closed cover $\left\{F_{m}: m \in \mathbb{N}\right\}$ of $B \cup Q$ and set $K_{m}=\Psi_{A}\left(F_{m} \cap\right.$ $\left.\bar{A}^{\uparrow \mathbb{N}}\right)$. Let $s \in S$ and $m$ be such that $K_{m} \cap\left\{q_{s^{\wedge} k}: k \in \mathbb{N}\right\}$ is infinite. Then for $\tilde{s} \in S$ such that $\tilde{s}(i)$ is the $s(i)$-th element of $A$ for each $i<|s|$, we have that $F_{m} \cap\left\{q_{\tilde{s}^{\wedge} k}: k \in A\right\}$ is infinite.

This completes the proof of Theorem 2.1. The following corollary of Theorem 2.1 answers in the negative Problem 15(1) of Bartoszyński and the second author [2].

Corollary 2.6. The union of a $\mathfrak{b}$-scale and $Q$ need not satisfy $\mathrm{S}_{1}(\Gamma, \Gamma)$.

\section{Reformulation For spaces of CONTINUOUS FUnCTIONS}

Let $Y$ be a (not necessarily metrizable) topological space. For $y \in Y$ and $A \subseteq$ $Y$, write $\lim A=y$ if $A$ is countable and an (any) enumeration of $A$ converges nontrivially to $y$. Let $\Gamma_{y}=\{A \subseteq Y: \lim A=y\}$. $Y$ has the Arhangel'skil property $\alpha_{2}[1]$ if $\mathrm{S}_{1}\left(\Gamma_{y}, \Gamma_{y}\right)$ holds for all $y \in Y$.

Fix a set of reals $X . C_{p}(X)$ is the subspace of the Tychonoff product $\mathbb{R}^{X}$ consisting of the continuous functions. It was recently discovered, independently by Bukovský and Haleš [4] and by Sakai [11, that $C_{p}(X)$ has the property $\alpha_{2}$ if, and only if, $X$ satisfies $\mathrm{S}_{1}\left(C_{\Gamma}, C_{\Gamma}\right)$.

Many additional connections of this type are studied in the literature. For families $\mathscr{A}$ and $\mathscr{B}$, consider the following prototype [12].

$\mathrm{S}_{\text {fin }}(\mathscr{A}, \mathscr{B})$ : For each sequence $\left\{\mathcal{U}_{n}\right\}_{n \in \mathbb{N}}$ of members of $\mathscr{A}$, there exist finite subsets $\mathcal{F}_{n} \subseteq \mathcal{U}_{n}, n \in \mathbb{N}$, such that $\bigcup_{n} \mathcal{F}_{n} \in \mathscr{B}$. 
For a topological space $Y$ and $y \in Y$, let $\Omega_{y}=\{A \subseteq Y: y \in \bar{A} \backslash A\}$. $Y$ has the Arhangel'skiur countable fan tightness [1] if $\mathrm{S}_{\mathrm{fin}}\left(\Omega_{y}, \Omega_{y}\right)$ holds for each $y \in Y$. $Y$ has the Reznichenko property if for each $y \in Y$ and each $A \in \Omega_{y}$, there are pairwise disjoint finite sets $F_{n} \subseteq A, n \in \mathbb{N}$, such that each neighborhood $U$ of $y$ intersects $F_{n}$ for all but finitely many $n$.

For sets of reals $X, C_{p}(X)$ has countable fan tightness and the Reznichenko property if, and only if, all finite powers of $X$ have the Hurewicz property $\mathrm{U}_{\text {fin }}(\mathcal{O}, \Gamma)$ [9. Thus, Theorem 2.1 can be reformulated as follows.

Theorem 3.1. Assume that $\mathfrak{b}=\mathfrak{c}$. There exists a set of reals $X$ without perfect subsets such that $C_{p}(X)$ has countable fan tightness and the Reznichenko property, but does not have the Arhangel'skiu property $\alpha_{2}$.

A topological space $Y$ has the Sakai strong countable fan tightness if $\mathrm{S}_{1}\left(\Omega_{y}, \Omega_{y}\right)$ holds for each $y \in Y$. Sakai proved that for sets of reals, $C_{p}(X)$ has strong countable fan tightness if, and only if, all finite powers of $X$ satisfy $\mathrm{S}_{1}(\mathcal{O}, \mathcal{O})$ [10. For sets of reals $X, C_{p}(X)$ has strong countable fan tightness and the Reznichenko property if, and only if, all finite powers of $X$ satisfy $\mathrm{U}_{\text {fin }}(\mathcal{O}, \Gamma)$ as well as $\mathrm{S}_{1}(\mathcal{O}, \mathcal{O})[8$.

If $\mathfrak{b} \leq \operatorname{cov}(\mathcal{M})$ and $X$ is a union of a $\mathfrak{b}$-scale and $Q$, then all finite powers of $X$ satisfy $\mathrm{U}_{\text {fin }}(\mathcal{O}, \Gamma)$ as well as $\mathrm{S}_{1}(\mathcal{O}, \mathcal{O})$ 2. As the Continuum Hypothesis (or just Martin's Axiom) implies that $\mathfrak{b}=\operatorname{cov}(\mathcal{M})=\mathfrak{c}$, we have the following.

Corollary 3.2. Even for $C_{p}(X)$ where $X$ is a set of reals, the conjunction of strong countable fan tightness and the Reznichenko property does not imply the Arhangel' skiu property $\alpha_{2}$.

\section{Concluding Remarks and open problems}

Our results are consistency results. What is not settled is whether the answers to the problems addressed in this paper are undecidable.

Problem 4.1. Is it consistent that all sets of reals that have the Hurewicz property $\mathrm{U}_{\text {fin }}(\mathcal{O}, \Gamma)$ but have no perfect subsets satisfy $\mathrm{S}_{1}(\Gamma, \Gamma)$ ?

Problem 4.2. Is it consistent that each union of a $\mathfrak{b}$-scale and $Q$ satisfies:

(1) $\mathrm{S}_{1}(\Gamma, \Gamma)$ ?

(2) $S_{1}(\Gamma, \Gamma)$ in all finite powers?

Problem 4.3. Is it consistent that for each set of reals $X$, if $C_{p}(X)$ has both strong countable fan tightness and the Reznichenko property, then $C_{p}(X)$ has the Arhangel'skil property $\alpha_{2}$ ?

\section{REFERENCES}

[1] A. V. Arhangel'skiı̌, The frequency spectrum of a topological space and the classification of spaces, Soviet Mathematical Doklady 13 (1972), 1186-1189. MR0394575 (52:15376)

[2] T. Bartoszyński and B. Tsaban, Hereditary topological diagonalizations and the MengerHurewicz Conjectures, Proceedings of the American Mathematical Society 134 (2006), 605615. MR2176030 (2006f:54038)

[3] A. R. Blass, Combinatorial cardinal characteristics of the continuum, in: Handbook of Set Theory (M. Foreman, A. Kanamori, and M. Magidor, eds.), Kluwer Academic Publishers, Dordrecht, to appear. http://www.math.lsa.umich.edu/ ablass/hbk.pdf

[4] L. Bukovský and J. Haleš, QN-spaces, wQN-spaces and covering properties, Topology and its Applications 154 (2007), 848-858. MR2294632 
[5] L. Bukovský, I. Reclaw, and M. Repický, Spaces not distinguishing pointwise and quasinormal convergence of real functions, Topology and its Applications 41 (1991), 25-41. MR.1129696 (93b:54037)

[6] W. Hurewicz, Über Folgen stetiger Funktionen, Fundamenta Mathematicae 9 (1927), 193204.

[7] W. Just, A. W. Miller, M. Scheepers, and P. J. Szeptycki, The combinatorics of open covers, II, Topology and its Applications 73 (1996), 241-266. MR1419798 (98g:03115a)

[8] Lj. D. R. Kočinac and M. Scheepers, Function spaces and a property of Reznichenko, Topology and its Applications 123 (2002), 135-143. MR1921655 (2003g:54036)

[9] Lj. D. R. Kočinac and M. Scheepers, Combinatorics of open covers (VII): Groupability, Fundamenta Mathematicae 179 (2003), 131-155. MR2029229 (2005c:54020)

[10] M. Sakai, Property $C^{\prime \prime}$ and function spaces, Proceedings of the American Mathematical Society 104 (1988), 917-919. MR964873(89m:54004)

[11] M. Sakai, The sequence selection properties of $C_{p}(X)$, Topology and its Applications 154 (2007), 552-560. MR2280899

[12] M. Scheepers, Combinatorics of open covers I: Ramsey theory, Topology and its Applications 69 (1996), 31-62. MR1378387 (97h:90123)

[13] B. Tsaban and L. Zdomskyy, Scales, fields, and a problem of Hurewicz, Journal of the European Mathematical Society, to appear.

Institute of Mathematics, Physics and Mechanics and Faculty of Education, UniverSity of Ljubluana, P.O.B. 2964, Ljubljana, Slovenija 1001

E-mail address: dusan.repovs@guest.arnes.si

Department of Mathematics, Bar-Ilan University, Ramat-Gan 52900, Israel; and Department of Mathematics, Weizmann Institute of Science, Rehovot 76100, Israel

E-mail address: tsaban@math.biu.ac.il

Kurt Gödel Research Center for Mathematical Logic, Währinger Str. 25, A-1090 Vienna, Austria

E-mail address: lzdomsky@gmail.com 